\title{
Local Radial Basis Function Collocation Method for Numerical Solutions of Sloshing Phenomenon
}

\author{
Chia-Ming Fan ${ }^{1, a}$ and Wei-Shiang Lai ${ }^{2, b}$ \\ ${ }^{1}$ Department of Harbor and River Engineering \& Computation and Simulation Center, National \\ Taiwan Ocean University, Keelung, Taiwan \\ ${ }^{2}$ Department of Harbor and River Engineering \& Computation and Simulation Center, National \\ Taiwan Ocean University, Keelung, Taiwan \\ acmfan@ntou.edu.tw, bdilonky@yahoo.com.tw
}

\begin{abstract}
Keywords: Meshless method, Sloshing, Local radial basis function collocation method, Moving-boundary problem.
\end{abstract}

\begin{abstract}
A promising domain-type meshless method is adopted to analyze the moving-boundary problems of sloshing phenomenon in a horizontally-excited numerical tank. When a tank partially filled with liquid is excited, the flow fields and the evolutionary profiles of free surface are known as the sloshing phenomenon. The local radial basis function collocation method is a newly-developed domain-type meshless method, so the time-consuming tasks of mesh generation and numerical quadrature can be avoided. The spatial derivatives at every node can be expressed as linear combination of nearby function values with different weighting, since the interpolation within every subdomain is achieved by function expansion of radial basis functions. Some numerical results and comparisons are provided to verify the merits of the proposed meshless scheme.
\end{abstract}

\section{Introduction}

To realize the fluid dynamics of the sloshing phenomenon is very important to us since the sloshing phenomenon can be used to describe various engineering problems, such as resonance in ship tanks in the ocean and the nuclear fuel storage pool oscillated during earthquake. In this paper, the flow field of sloshing phenomenon is considered to be the potential flow, so the governing equation is the Laplace equation for velocity potential. In the past, many numerical schemes have been adopted to accurately analyze the non-breaking waves in the sloshing problems [1-3]. Though some numerical schemes have been proposed to deal with the sloshing problems, we proposed an accurate and simple numerical scheme to efficiently and accurately simulate the flow field of sloshing problems in a horizontally-excited tank.

We adopted the explicit Euler method and the semi-Lagrangian approach [3] to handle two free-surface boundary conditions. Because the computational domain and the profile of free surface change at every time step, the local radial basis function collocation method (LRBFCM) [4-6] is used to efficiently deal with the potential problem with mixed boundary conditions at every time step. Recently, various meshless methods have been proposed to analyze engineering applications in order to avoid the time-consuming tasks of mesh generation and numerical quadrature. The LRBFCM is one of the most-promising domain-type meshless methods. Besides, the localization in the LRBFCM can alleviate the problems of ill-conditioning matrix and form a system of sparse matrix. Recently, many researchers [4-6] have successfully applied the LRBFCM to analyze various engineering problems. Thus, we adopted the LRBFCM for spatial discretization of the potential problems of the sloshing phenomenon at every time step.

\section{Mathematical Problems}

Two coordinate systems are adopted at the same time. One is the inertial Cartesian coordinate system $(X, Z)$, used to describe the spatial positions of the considered tank, $X=X_{T}(t)$. The other one is the Cartesian coordinate system $(x, z)$, which is attached to the left bottom corner of this tank. The 
considered rectangular tank is depicted in Fig. 1. Since the fluid flow in the sloshing problem is potential flow, the velocity potential is governed by the Laplace equation,

$$
\nabla^{2} \phi=0, \quad(x, z) \in \Omega,
$$

where $\phi(x, z, t)$ is the velocity potential. $\Omega$ is the computational domain and it will deform due to the evolutionary profile of free surface. In addition, the impermeable boundary condition is given along bottom and solid wall,

$$
\left.\frac{\partial \phi}{\partial z}\right|_{z=0}=\left.0 \quad \& \quad \frac{\partial \phi}{\partial x}\right|_{x=0, b}=0
$$

The dynamic and the kinematic free-surface boundary conditions are given along the free surface,

$$
\left.\frac{\partial \phi}{\partial t}\right|_{z=\zeta+h}=-g \zeta-\frac{1}{2}\left[\left(\frac{\partial \phi}{\partial x}\right)^{2}+\left(\frac{\partial \phi}{\partial z}\right)^{2}\right]-\left.x X_{T}^{\prime \prime}(t) \& \frac{\partial \zeta}{\partial t}\right|_{z=\zeta+h}=-\frac{\partial \phi}{\partial x} \frac{\partial \zeta}{\partial x}+\frac{\partial \phi}{\partial z}
$$

where $\zeta$ is the free surface elevation, measured above still water level. $g$ is the acceleration due to gravity and $X_{T}^{\prime \prime}(t)$ is the horizontal accelerations of the considered tank.

\section{Numerical Methods}

We re-write the dynamic and the kinematic free-surface boundary conditions by following the semi-Lagrangian approach [3] and use the explicit Euler method to discretize them,

$$
\begin{aligned}
& \phi^{n+1}=\phi^{n} \\
& +\Delta t\left\{-g \zeta^{n}-\frac{1}{2}\left[\left(\frac{\partial \phi^{n}}{\partial x}\right)^{2}+\left(\frac{\partial \phi^{n}}{\partial z}\right)^{2}\right]+\frac{\partial \phi^{n}}{\partial z}\left(\frac{\partial \phi^{n}}{\partial z}-\frac{\partial \phi^{n}}{\partial x} \frac{\partial \zeta^{n}}{\partial x}\right)-x^{n} X_{T}^{\prime \prime}\left(t^{n}\right)\right\} \\
& \zeta^{n+1}=\zeta^{n}+\Delta t\left\{\frac{\partial \phi^{n}}{\partial z}-\frac{\partial \phi^{n}}{\partial x} \frac{\partial \zeta^{n}}{\partial x}\right\}
\end{aligned}
$$

where $\triangle \mathrm{t}$ is the time increment. The superscripts $n$ and $n+1$ describe the physical values at the $n^{\text {th }}$ and the $(n+1)^{\text {th }}$ time steps, respectively. The velocity potential and the elevation along free surface at the $(n+1)^{\text {th }}$ time step can be directly acquired from Eqs. 4-5. According to the vertical movements of free surface, the nodes inside computational domain will move proportionally and vertically. In consequence, a Laplace problem of the velocity potential at the $(n+1)^{\text {th }}$ time step is yielded.

While a sub-domain at the $i^{\text {th }}$ node is considered, the $n_{s}$ nearest nodes around the $i^{\text {th }}$ node will be found and the sub-domain of the $i^{\text {th }}$ node is formed. The solution within the sub-domain is expressed as the linear combination of radial basis functions,

$$
w(x, y)=\sum_{j=1}^{n_{s}} \beta_{j} \Phi\left(r_{j}\right)
$$

where $j$ is the local index in the sub-domain. $r_{j}=\left|\vec{x}-\vec{x}_{j}^{i}\right|$ is the distance between nodes in the sub-domain. $\left\{\beta_{j}\right\}_{j=1}^{n_{s}}$ are the unknown coefficients. $\Phi\left(r_{j}\right)$ is the radial basis function and we used the multiquadrics function $\left(\Phi\left(r_{j}\right)=\sqrt{1+b r_{j}^{2}}\right)$ in this paper, where $b$ is the shape parameter. Because every node in this sub-domain should satisfy the interpolation equation, the interpolation at every node in the sub-domain can yield the following system of linear equations,

$$
\{w\}=[A]\{\beta\} \text {, }
$$


where $\{w\}=\left[w_{1}^{i}, w_{2}^{i}, w_{3}^{i}, \ldots \ldots, w_{n_{s}}^{i}\right]^{T}$ and $\{\beta\}=\left[\beta_{1}, \beta_{2}, \beta_{3}, \ldots \ldots ., \beta_{n_{s}}\right]^{T}$ denote the nodal values of the nodes inside the sub-domain and the unknown coefficients.

While the second-order derivative with respect to $x$ at the $i^{\text {th }}$ node is needed, the derivative can be expressed by directly differentiating Eq. 6 with respect to $x$,

$$
\left.\frac{\partial^{2} w}{\partial x^{2}}\right|_{i}=\sum_{j=1}^{n_{s}} \beta_{j} \frac{\partial^{2} \Phi\left(\left|\vec{x}_{i}-\vec{x}_{j}^{i}\right|\right)}{\partial x^{2}}=\left\{\frac{\partial^{2} \Phi_{i, j}}{\partial x^{2}}\right\}^{T}\{\beta\}=\left\{\frac{\partial^{2} \Phi_{i, j}}{\partial x^{2}}\right\}^{T}[A]^{-1}\{w\}
$$

where $\left\{\frac{\partial^{2} \Phi_{i, j}}{\partial x^{2}}\right\}=\left\{\frac{\partial^{2} \Phi_{i, 1}}{\partial x^{2}}, \frac{\partial^{2} \Phi_{i, 2}}{\partial x^{2}}, \frac{\partial^{2} \Phi_{i, 3}}{\partial x^{2}}, \ldots . ., \frac{\partial^{2} \Phi_{i, n_{s}}}{\partial x^{2}}\right\}^{T}$

is the vector of second-order derivative of radial basis function with respect to $x$. Similarly, these above-described procedures can be implemented at every node to acquire the expressions of any-order spatial derivative. We use these expressions of spatial derivatives at every node to form a system of linear algebraic equations by enforcing the satisfactions of governing equation at every interior node and boundary condition at every boundary node. Once the resultant system is solved, the numerical results at the present time step are acquired and the numerical procedures can march to next time step.

\section{Numerical Results and Comparisons}

This example is the moving-boundary problem of sloshing phenomenon in a rectangular tank, subjected to horizontal excitation. The length of the tank and depth of initially still fluid are 0.9 and 0.6, respectively. The fluid in the considered tank is still at first and then the rectangular tank is forced to oscillate horizontally by the following acceleration,

$$
X_{T}^{\prime \prime}(t)=-a_{h} \omega_{h}^{2} \sin \left(\omega_{h} t\right), \quad t \geq 0,
$$

where the amplitude $\left({ }^{a_{h}}\right)$ and the horizontal radial acceleration $\left(\omega_{h}\right)$ are $a_{h}=2 \times 10^{-3}$ and $\omega_{h}=5.5$, respectively. The initial conditions for elevation of free surface and velocity potential in the entire domain are zero. The following parameters are adopted: number of total nodes $(N=961)$, time increment ( $\triangle t=0.001)$ and number of nodes in sub-domain $\left(n_{s}=15\right)$. The evolutionary profiles of the elevations at the right-end node of free surface are shown in Figs. 2(a)-2(b) and are compared well with the numerical results by the Trefftz method [2] and the finite element method [2].

From the provided comparisons in Figs. 2(a)-2(b), it is obvious that the numerical solutions by adopting different numbers of total nodes and different time increments are almost the same. Furthermore, the amplitude and the horizontal radial acceleration in Eq. 9 are changed to $a_{h}=1 \times 10^{-2}$ and $\omega_{h}=4.5$. In the second test, the following parameters are used: $N=1681, \Delta t=0.001$, and $n_{s}=15$. The evolutionary profiles of elevations at the right-end node and the left-end node of free surface are demonstrated in Figs. 3(a)-3(b) and the numerical results are also compared well with other numerical solutions [2]. From the provided results and comparisons, it could be deduced that the proposed meshless numerical method, based on the LRBFCM, has satisfying accuracy, stability and consistency while two-dimensional sloshing problems in a horizontally-excited tank are considered.

\section{Conclusions and Discussions}

In this paper, we proposed a meshless numerical method to analyze the moving-boundary problems of sloshing phenomenon in a horizontally-excited numerical tank. The explicit Euler method and the semi-Lagrangian scheme are adopted for integrating two nonlinear free-surface boundary conditions, while the LRBFCM is used to efficiently solve the potential problem at every time step. The LRBFCM is free from mesh generation and numerical quadrature, so to adopt the 
LRBFCM to analyze the sloshing problems is very efficient. The provided numerical results are compared very well with other numerical solutions. Besides, the results by using different numbers of nodes and different time increments are provided to validate the stability and consistency of the proposed method.

\section{References}

[1] J.B. Frandsen: J. Comput. Phys. Vol. 196 (2004), p. 53.

[2] E. Kita, J. Katsuragawa and N. Kamiya: Eng. Anal. Bound. Elem. Vol. 28 (2004), p. 677.

[3] X.T. Zhang, B.C. Khoo and J. Lou: Ocean Eng. Vol. 33 (2006), p. 2310.

[4] H.F. Chan and C.M. Fan: Numer Heat Tranf. B-Fundam. Vol. 63 (2013), p. 284.

[5] C.M. Fan, C.S. Chien, H.F. Chan and C.L. Chiu: Int. J. Heat Mass Transf. Vol. 57 (2013), p. 500.

[6] G. Kosec and B. Sarler: CMES-Comp. Model. Eng. Sci. Vol. 25 (2008), p. 197.

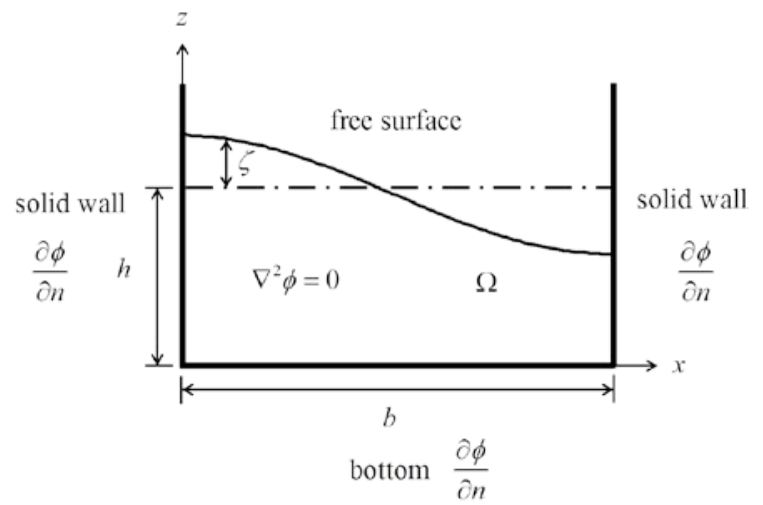

Figure 1. The schematic diagram of a horizontally-excited numerical tank.

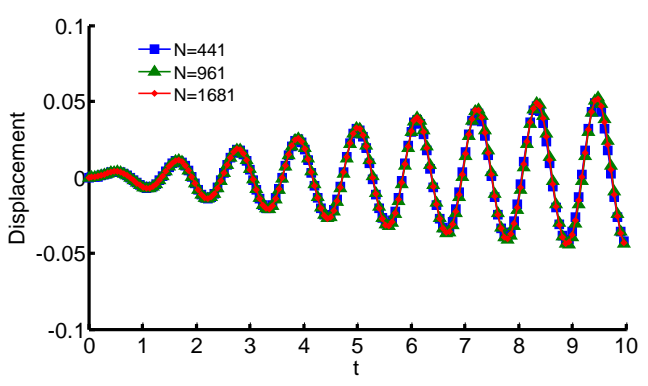

(a)

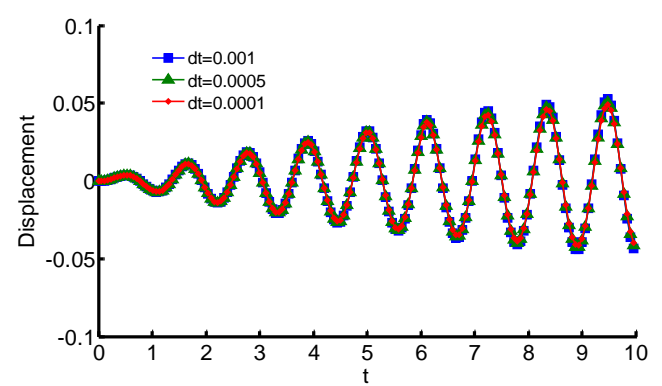

(b)

Figure 2. The evolutionary profiles of elevation at the right-end node of free surface by using (a) different numbers of nodes and (b) different time increments.

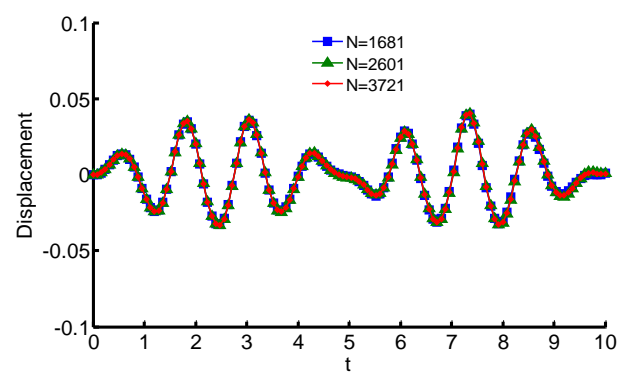

(a)

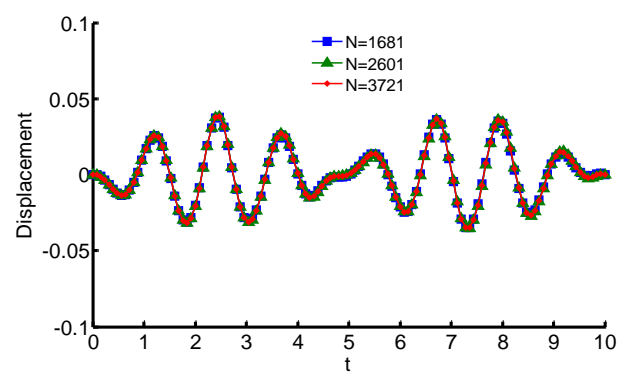

(b)

Figure 3. The evolutionary profiles of elevation at (a) right-end node and (b) left-end node of free surface by using different numbers of nodes. 